\title{
THE DISINFECTANT ACTION OF CERTAIN BACTERIAL STAINS.*
}

\author{
A NDREW M. JANSEN. $\dagger$ \\ (From the Bacteriological Laboratory of the Ohio State University.)
}

\section{HISTORICAL.}

Among the earliest workers to note the disinfectant action of bacterial stains were Cornil and Babes, ${ }^{,}$two botanists, who found, in working with methyl violet, that it possessed a toxic action on various bacteria.

In 1890 Stilling published results of work with a dye, probably a mixture of dyes which he called pyoktannin. His experiments, none too carefully performed, with $B$. subtilis, $B$. anthracis, and $M$. aureus, led him to the conclusion that the dye was a general bacterial poison, its antiseptic strength being three times that of bichlorid of mercury, and that the action of the dye was directly proportional to the strength of the dilution used, and to the time of the exposure.

Spina $^{2}$ in his article speaks of the use of indigo and methylene blue in the staining of culture media. He uses, however, in roo c.c. of culture media only a few drops of a sterile saturated solution of the dye, this, of course, being too high a dilution to have any active disinfectant or bactericidal action, even if the stain itself possessed such a property. Spina also notes that in this dilution bacteria have a marked reducing action upon the stain used.

Huber $^{3}$ shows the effect of diffused light upon broth cultures of streptococci stained with eosin and erythrosin and exposed to the light for different periods of time as follows: Streptococcus exposed to diffused light for $\mathbf{I} \mathrm{hr}$. showed growth on first and second day in unstained broth, eosin stained broth, and erythrosin stained broth. Exposure for 3 and $6 \mathrm{hrs}$. resulted in growth on first and second day in unstained broth, but no growth in eosin stained broth or erythrosin stained broth. The control which was not exposed showed growth in all 3 broths. Results with $B$. diphtheriae were practically the same except that there was only a slight growth in the 3 -hr. exposure on the second day.

Conradi and Drigalskit deal principally with crystal violet in culture media which inhibited numerous cocci and bacteria, but had no effect upon B. coli and B. typhosus. A number of organisms belonging to the subtilis group are also mentioned among those that grew on their crystal violet plates.

Penzolds also notes a definite bactericidal action of certain bacterial stains.

Another work of interest and of more recent date is that of DeWitt. ${ }^{6}$ In these experiments with the various dyes, two drops of a I per cent solution of each dye are

* Received for publication August 4, 19:3.

$\dagger$ Dr. Andrew M. Jansen died of an infection of glanders on January 4, I9I4.

I Mitt., Strassburg, 189o. $\quad 3$ Arch. f. Hyg., 1905, 54, p. 53.

2 Centralbl.f. Bakteriol., I887, Ir, p. 7r. 4 Zischr. f. Hyg., I902, 5, p. 283.

s Arch. f. exper. Pathol. u. Pharmakol., 1890, 26, p. 310.

- Jour. Infect. Dis., 19r3, r 2, p. 68. 
used to each tube of agar, and the growth of the various bacteria noted upon the corresponding plate, particular attention being given to the effect of the dye upon culture media.

Much work has been done, especially in the last few months, upon the inhibitive and selective action of certain of the anilin dyes upon certain bacteria in culture media. Churchman ${ }^{x}$ shows clearly that the growth of most of the gram-positive organisms such as $M$. aureus, $B$. anthracis, etc., is greatly inhibited when gentian violet is added to the media in the proportion of $\mathrm{I}-100,000$, while the growth of the majority of gram. negative organisms such as $B$. coli, $B$. typhosus, shows little or no diminution in luxuriance.

In the following experiments, an effort was first made to determine the disinfectant action of the dye itself, without any of the common constituents, alcohol, anilin, etc., which are used when the stain is employed in regular laboratory routine work. The first dye considered was Merck's methylene blue.

Dilutions of the dye were made in sterile water as follows, $I-200, I-500,1-1,000$, $\mathrm{I}-2,000$, and $\mathrm{I}-10,000$. The first organism used was a 24 -hour broth culture of $B$. coli recently isolated from feces. Ten cubic centimeters of each of the above dilutions were placed in five sterile test tubes with a sterile pipette. One-half cubic centimeter of the broth culture of $B$. coli was placed in each of the dilutions, shaken up well, and agar plates made from the dilutions at intervals of $3,5,10$, and 30 mins. In the I-I0,000 dilution the intervals were $5,15,30$, and 60 mins. each. In making the plate one loopful of the mixture-the dilution to which 0.5 c.c. of broth culture had been added - was transferred to a melted agar tube well shaken, and plate poured as usual. In this manner the amount of stain carried over on the needle would be so small, especially when diluted by the agar content of the tube, that it would have no appreciable inhibitive action.

The plates were incubated at $37^{\circ} \mathrm{C}$. for $48 \mathrm{hrs}$. before examination. The agar used was ordinary meat extract agar, standardized to $\mathrm{I}$ per cent acid to phenolphthalein. B. coli exposed for 3,5 , Io, and $3 \circ$ mins. to methylene blue in dilutions of $\mathrm{I}-200, \mathrm{I}-500, \mathrm{I}-\mathrm{I}, 000$, and $\mathrm{I}-2,000$ showed growth. In dilution of $\mathrm{I}-10,000 \mathrm{~B}$. coli showed growth in $5,15,30$, and 60 mins.

No disinfectant action was manifested by the stain, there being only a slight inhibition in the $1-200$ and $I-500$ dilutions exposed for 30 mins. The $1-10,000$ dilution, exposed for one hour, also showed a slight decrease in the number of colonies on the plate.

In the next experiment, a culture of $M$. aureus recently isolated from a boil was used. The same stain, dilutions, periods of exposure, and technic were used as in the preceding, except that the 24 -hour broth culture of the coccus was filtered through sterile filter paper to remove all clumps. The stain had no bactericidal action whatever, the results being about the same as when $B$. coli was used.

Gentian violet was next used in the same dilutions and with the same periods of exposure. B. coli and $M$. aureus were used as with methylene blue. B. coli was not inhibited appreciably in growth, even in the low dilutions of $\mathrm{I}-200$ and $\mathrm{I}-500$, except

Jour. Exper. Med., I91 2, I6, p. 22r. 
in the ro- and 30-minute exposures, and that of $1-1,000$ for 30 mins. In the higher dilutions, little inhibition was shown even in the longer exposures.

A marked disinfectant action was shown with the staphylococcus. Inhibition was complete in dilutions up to $I-2,000$ at even the shortest exposure, 3 mins. In the I-IO,000 dilution a few colonies were found in the $5^{-}$and ${ }_{15}$-min. exposures, but none in the 30- and 60-min. exposures, showing that this dilution would have a complete inhibitive action in culture media.

Crystal violet showed about the same selective action as gentian violet, except that $B$. coli showed more colonies in the $30-\mathrm{min}$. exposure, $\mathrm{I}-200$ dilution, of crystal violet, than gentian violet; the inhibitive action on $M$. aureus was about the same in each stain.

The effect of dilutions of a sterile aqueous solution of fuchsin was then tried on the same organisms. The lowest dilution used was $1-500$, as fuchsin proved to be insoluble in water in the proportion of $\mathrm{I}-200$.

No disinfectant or selective action is manifested upon either $B$. coli or $M$. aureus with this stain. In the dilutions of $\mathrm{I}-500$ and $\mathrm{I}-\mathrm{I}, 000$ for $30 \mathrm{mins}$. there seems to be a slight inhibitive action, the $30-\mathrm{min}$. exposure plate of $B$. coli ${ }_{1}-500$ dilution showing no growth.

In the next series of experiments an effort was made to find the disinfectant action of the stains as prepared and used in the laboratory, and containing the usual constituents, such as alcohol, phenol, anilin, etc.

Merck's methylene blue was first used, made up as follows: $3 \circ$ parts of saturated alcoholic solution of methylene blue and 100 parts of $\mathrm{NaOH}(\mathrm{I}-10,000)$. In sterile test tubes were placed ro c.c. of this stain, and 0.5 c.c. of a 24 -hour broth culture of each organism used was added to the stain in the test tube, well shaken, and exposed for the following intervals (agar plates were made by taking one loopful from the mixture of the stain and broth culture at the end of each interval): I, 3, 10, 30, and 60 mins., respectively. Plates were made on ordinary extract agar as before, and incubated at $37^{\circ} \mathrm{C}$. for $48 \mathrm{hrs}$. The organisms used were laboratory stock cultures as follows: $B$. coli, isolated from feces; $M$. aureus, isolated from a boil; B. typhosus, isolated in laboratories of the University of Chicago; B. anthracis; B. mallei, from the University of Chicago; pneumococcus (Winslow No. 80, American Museum of Natural History). No active disinfectant action was shown by the stain even in the I-hr. exposures. The pneumococcus and mallei plates contained only a few colonies in the Io-min., 30-min., and I-hour exposures. The stain as used contained about 22 per cent of alcohol. This dilution, as has been shown by previous experiments, has little or no bactericidal action, and as shown by comparing the last series with the one in which the aqueous solution was used, does not add anything materially to the disinfectant action of the stain.

Anilin gentian violet made up for staining as follows was then used: I part of saturated alcoholic solution of gentian violet and io parts of anilin water.

Anilin water was made up by using 4 parts of freshly distilled anilin to Ioo parts of distilled water. The same organisms, dilutions, and technic were used as in the preceding experiment. Growth was inhibited in each case with anilin gentian violet. 
Carbolfuchsin was . 冫, made up for staining as follows: I part of saturated alcoholic solution of fuchsin and to parts of 5 per cent phenol.

CARBOLFUCHSIN (as for staining).

Period of Exposure.

\begin{tabular}{|c|c|c|c|c|c|}
\hline Organism & I $\min$. & 3 mins. & ro mins. & 30 mins. & $\mathrm{r}$ hr. \\
\hline 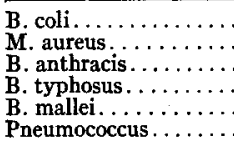 & $\begin{array}{c}+++ \\
++ \\
+++ \\
++ \\
++ \\
+\end{array}$ & $\begin{array}{c}++ \\
++ \\
+++ \\
++ \\
++\end{array}$ & $\begin{array}{c}++ \\
+ \\
++ \\
\pm \\
\pm\end{array}$ & $\begin{array}{l}\frac{t}{t} \\
\pm \\
-\end{array}$ & $\begin{array}{l} \pm \\
\pm \\
\bar{z}\end{array}$ \\
\hline
\end{tabular}

+ indicates growth in varying degree; - indicates no growth.

As the aqueous solutions of this stain used in the previous experiments showed little or no bactericidal action, the disinfectant strength of the stain shown in this experiment is undoubtedly due to the percentage of phenol contained. The I-hour plate of $B$. anthracis showed only a few colonies, probably the result of spores which resisted the action of the disinfectant content of the stain for that period.

In the next work, an effort was made to determine whether some of the ordinary bacteria cultivated in the laboratory are alive and capable of reproduction after they have been subjected to the regular laboratory technic of staining.

Glass cover slips were used as follows: The cover slip was held in a holder which prevented its touching the table or any surrounding object and passed through a flame until completely sterile, and then allowed to cool. After cooling, a loopful of a 24-hour broth culture of the organism was placed on the slip, allowed to dry, and fixed in the usual manner by passing quickly through a flame three times. The slip was then stained in the prescribed manner, depending upon the stain used, the stain washed off with sterile water and the slip dropped into a sterile petri dish with the stained side up. A melted agar tube was then poured in the usual manner, covering the slip. The plates were incubated at $37^{\circ} \mathrm{C}$. for $48 \mathrm{hrs}$. before examination. Control plates were run on slips containing the organism, dried and fixed, but unstained, in order to obtain an idea as to the effect of drying and fixing upon the bacteria.

In the following experiments $24-\mathrm{hr}$. broth cultures were stained with methylene blue and anilin gentian violet for I min., and with carbolfuchsin for io secs. Growth occurred in the controls unless otherwise specified. B. anthracis, M. aureus, and Pseudomonas pyocyanea showed growth in methylene blue and carbolfuchsin, but not in anilin gentian violet or Gram stain. In B. typhosus, B. coli 
isolated from feces, $M$. melitensis, and B. enteritidis, growth occurred after methylene blue staining but carbolfuchsin, anilin gentian violet, and Gram stain inhibited growth. B. diphtheriae, B. cholerae gallinarum, Sarcina tetragena, and Microspira comma showed no growth, but since the control plates with one exception were sterile, the organisms were probably killed by heating and drying.

\section{SUMMARY.}

The study of the antiseptic action of the various dyes in the foregoing experiments warrants the following statements:

I. Aqueous solutions of methylene blue or fuchsin have little or no bactericidal action, even in dilutions as low as $1-200$ and $I-500$.

2. Aqueous solutions of gentian violet have a marked disinfectant action on the gram-positive organisms used in as high dilutions as I-I0,000 exposed for 30 mins. only. In the case of the gram-negative $B$. coli a dilution of $\mathrm{I}-500$ for $30 \mathrm{mins}$. was required to show any decided bactericidal action. Crystal violet showed about the same selective and bactericidal action as gentian violet.

3. Methylene blue as made up for staining showed little or no bactericidal action, the percentage of alcohol contained being too low.

4. Anilin gentian violet as made up for staining in the laboratory showed a strong and decided disinfectant action, the r-min. exposures being sufficient to kill all the organisms used, the gramnegatives as well as the gram-positives.

5. Carbolfuchsin as made up for staining proved also of some value as a bactericidal agent, the action, of course, being almost entirely due to the phenol content.

6. In the cover slip experiments it was rather difficult to determine in some cases whether the disinfectant action was due to the drying and fixing, or to the stain itself. Controls of dried and fixed but unstained organisms were accordingly run. It was found in general that if the organisms in question survived drying and fixing, little effect was seen from the use of methylene blue. Many were killed by carbolfuchsin, and all by anilin gentian violet and Gram stain. 
Most pathogenic laboratory organisms which were stained with methylene blue in the ordinary way, if they survived drying and fixing, were alive, capable of reproduction, and would probably not be safe to handle without further sterilization.

All of the pathogenic organisms except spore forms when stained with carbolfuchsin (not dilute) might be handled with safety.

All of the ordinary pathogenic laboratory organisms may be safely handled after being stained with anilin gentian violet for I min., or the regular Gram stain. 